\title{
Design and Implementation of Local Traffic Scheduling Simulation System
}

\author{
Shoujin Wang ${ }^{1, a,}$ Chao Zhang ${ }^{1, ~ b, ~ M i n g ~ Z h a o ~}{ }^{1, c^{*}}$
}

School of Information \& Control engineering, Shenyang Jianzhu University, Shenyang, China

a 23917240@qq.com, b356396059@qq.com, c1094301006@qq.com

*The Corresponding author

Keywords: Local traffic scheduling; Simulation system; Intelligent transportation; Automatic demonstration

\begin{abstract}
Transportation is an indispensable part of the city, however, with the development of social economy, traffic congestion, traffic accidents, resource shortage and other related issues have become the common problem faced by all countries in the world. Selecting an optimal trafficscheduling scheme to alleviate the traffic congestion, reduce travel time, improve the environment and reduce resources is the significance of this system, in order to reduce the overall travel energy demand and save huge amount energy of for society. This system is developed with VS2010 and C\#.
\end{abstract}

\section{Introduction}

With the development of social economy, traffic congestion, traffic accidents and traffic related problems such as shortage of resources has become the common problem facing by the world. The number of small cars in China increased by nearly three times as much in only five years. The development of the automobile industry has brought convenience for people's work and life, and also triggered a series of traffic problems[1-2]. A large number of traffic lights as supporting facilities are built to deal with the traffic problems. Although these urban roads and various supporting facilities had improved a lot to a certain extent, the irrationality of traffic light design and scheduling had worsen the continuous traffic load growth, resulting in problems such as traffic congestion, transport capacity reduction, in order to cause traffic accidents.

Road traffic problem has become the bottleneck of urban economic development. There are two ways to solve road traffic problems: one is to build more roads in space to alleviate traffic pressure, the other is to build traffic control system based on time, improve the traffic capacity for the existing roads[3]. In the two methods, the demand of building new road system covers both huge investment and a lot of arable land. Meanwhile, it is more and more difficult to relocate large amount of residents for the existing urban road reconstruction[4-5]. Nevertheless, there is an obvious effect in building intelligent transportation system to solve the main road traffic congestion in big cities.

In this context, from the viewpoint of system, considering of vehicle and roads together, the idea of solving road traffic problems can be generated by applying kinds of high-tech technology, which is called intelligent transportation system[6]. Intelligent transportation system is to apply the advanced information technology, data communication transmission technology, electronic control technology and computer technology, network technology and other high and new technology to the transportation management system effectively, cooperate the person, vehicle, road closely.

\section{The Purpose and Meaning of the Research}

The main problems of current traffic include: First, frequent traffic accidents, which causes great threat to human life security. Second, severe traffic congestion, which increases the travel time and the energy consumption[7]. Third, the degree of air pollution and noise pollution is becoming more and more serious. 
Aiming at the problems above, this system will generate traffic simulation prediction according to the initial input conditions, and demonstrate on the screen in real time, make a evaluation to the performance of the traffic signal scheduling scheme, so as to find out the most suitable traffic scheduling scheme for the current road, reduce the traffic efficiency loss from setting traffic lights blindly, alleviate traffic congestion and reduce the number of traffic accidents and increase traffic safety; to ease traffic congestion and improve the efficiency of the traffic; improve the efficiency of the bus, to reduce the traffic load; reduce the pollution and save the energy[8].

The significance of this system is to choose an optimal traffic scheduling scheme, so as to relieve traffic congestion situation, reduce travel time, improve the environment to save resources, reduce the overall travel demand for energy; save a lot of energy for the society and reduce the cost of pollution control; Traffic noise is the main source of urban noise[9]. After ITS implementation, the speed of the vehicle change frequency and the number of parking are reduced, thus decrease noise pollution caused by the traffic; Intelligent traffic can make the full scope to the actual capacity of existing roads, increase road network utilization, decrease the number of road construction in road network planning and save the land resources occupied by road building.

\section{The System Design}

Road information generated simulation road input intersection. Including the length of the road, lane number the speed limit, etc. In order to apply to all kinds of road intersection, regardless of the actual road, each outer branch is taken as one road. The system architecture see fig. 1 .

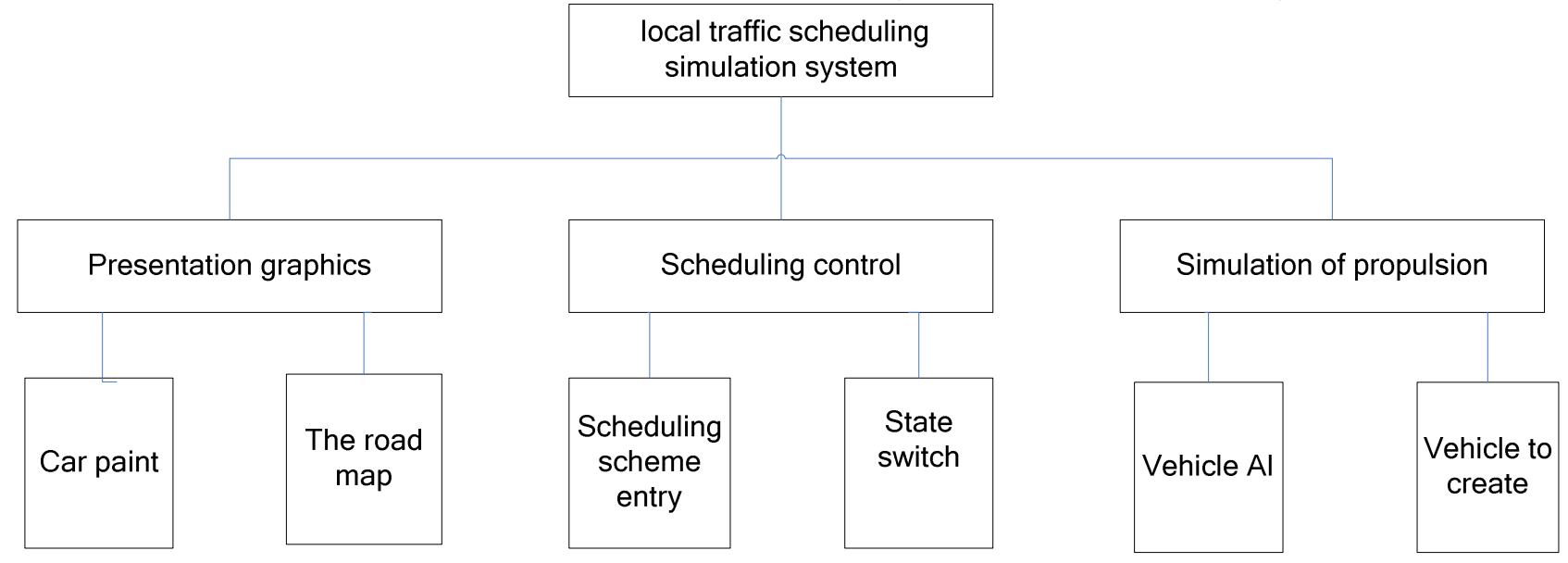

Figure 1. Finite the system architecture

Entering vehicle information to simulate the vehicle's random access. Including the route of the vehicle, speed control, the time interval, etc. The input signal control information (several states) is to control the connectivity between the roads. There is only one state at the same time, each state includes all connectivity between roads, which is stored in matrix. After entering the initial condition, system is able to demonstrate traffic condition according to the requirement manually or automatically, so as to achieve design goal.

Use Case Design. Use Case diagram is to point to by actors (Actor), Use Case (Use Case) and the relationship between them is used to describe the system function of the static view. Use Case diagrams (User Case) is known as the participants of the external users can observe system function model diagram, presents some participants and some cases, as well as the relationship between them. It is mainly used for on the function of the system, subsystem, or class behavior modeling.

The user has two use cases, input and parameter settings. There are two cases in scheme input, vehicle attribute input and input attribute. Parameter setting contains three settings as time setting, character setting and state setting.

Class Diagram Design. Class diagram is to display the static structure of the model, especially the class in models, the internal structure of Class, and their relationships with other classes, etc. Class diagram does not display the temporary information. Class diagrams are made up of many 
declarative model elements, such as Class, package, and their relationships, these elements and their contents are connected to each other. Class diagram can be organized in package, only shows the specific package of related content. Class diagram is the most commonly used UML diagram, display classes, interfaces, and the static structure and the relationships between them. It is used to describe structural design of the system. The most basic element of the Class diagram is Class or interface. The system class diagram see fig. 2 .

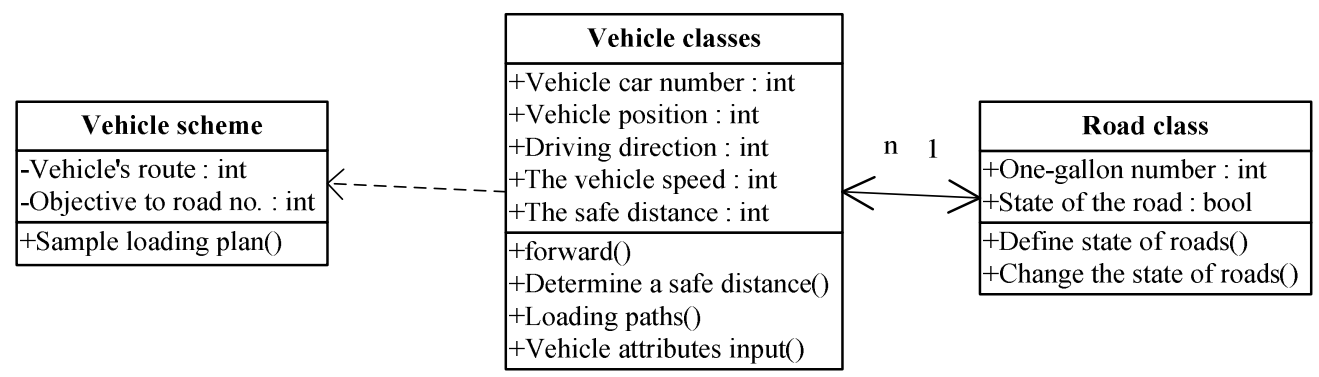

Figure 2. Finite system class diagram

In the system, class diagram is divided into three categories: system class, vehicle class and road class. Two properties in vehicle plan class: vehicle route attribute and aimed car number attribute. Five attributes in vehicle class: car number, vehicle location, driving direction, speed of vehicles and safe distances. Two attributes in road class: road numbers and road condition attributes. Vehicle class is depended on vehicle class; Road class and vehicle class are in correlation.

\section{System Sequence Diagram Design}

This system operation sequence is as follows: first, the user clicks the initialize button to initialize the program. The initialized information is sent to the control area. The control area will initialize information response to real-time demonstration area and information demonstration area. When the user simulate by entering vehicle simulation schemes, the information is passed to the real-time demonstration area through controlled area, start drawing the demonstration area according to the plan, display information in information presentation area. The system sequence diagram is shown in fig. 3 . 


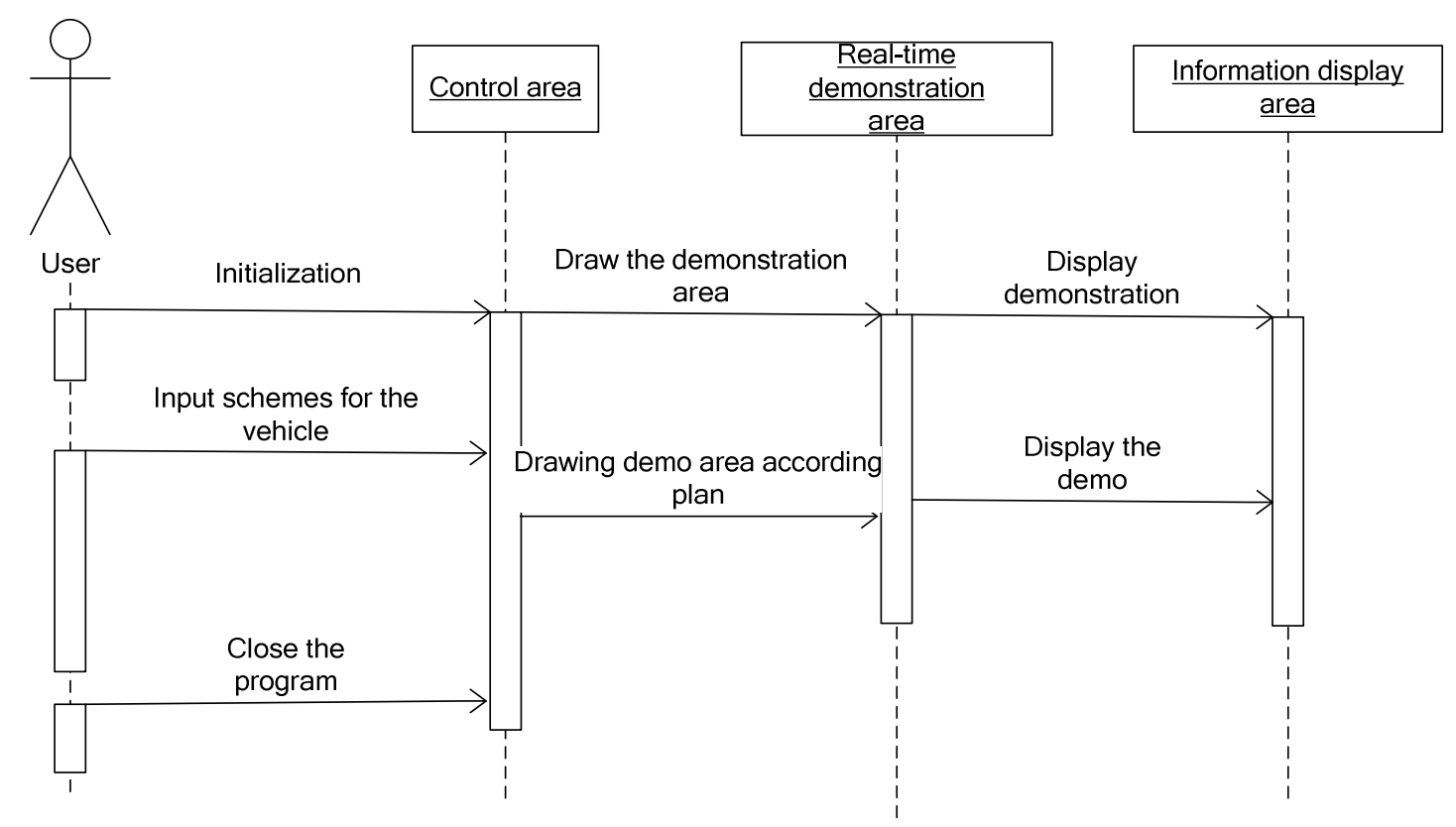

Figure 3. Finite system sequence diagram

\section{System Implementation}

Define the Vehicle Object. Create a Car class to define the vehicle object in the solution, defining vehicle path, including multiple starting points and end points, define the path array, path number, current path, the local car id, previous car id, next car id, car position, car direction, road id, destination id, current speed and maximum speed. An AI to accelerate or decelerate automatically through calculating distance between vehicles and vehicle speed and safe distance, as well as the path request processing.

Vehicle Random Create. Create an AutoCar Class in solution to store and create the vehicle randomly and enter into the simulated road. It will simulate according to the pre-set vehicle. With automatic control, the system will create vehicle in a certain random floating range.

Road Design. Create a Road Class to store the path information, which contains an array for accepting call and changing state. Each state can be interpreted as whether all roads can be directly connected, which is equivalent to the generalized traffic signal control. State is divided into $0 \mathrm{~s}$ and $1 \mathrm{~s}$ two; Roads are divided into $0,1,2,3,4$, representing four road direction, south, east, north and west. When the state is 0 , road 0,2 (south and north) are green, vehicles can pass. When the state is 1 , road 1,3 (east, west) are green, vehicles can pass. At the same time, each road vehicles turning right can pass.

The Automatic Control. Create a Timer Control Class for automatic switching status, for simulate road signals changing automatically. The switching time is controllable, are free to choose whether to open this function.

Interface UI Features. This system user interface is divided into three regions: information display area, real-time demonstration area, control area. Real-time demonstration area is realized by the control Panel. Roads, traffic lights and vehicle are created according to the code. Read the vehicle state in memory, and map to the main interface of the plot. Control area is mainly divided into the vehicle to create regional, advanced control, time advance, state transition and initialization area. Vehicles create area is responsible for the input of the five attributes and create options. Advanced automatic control area includes time, state automatic conversion, automatic simulation vehicles pushed into three automatic control options, and presentation speed control. When time automatic propulsion option is checked, the system will automatically advance time, the next step for vehicle operation. In the same way, with the state of automatic conversion, automatic simulation vehicle automatic conversion after check the road condition and automatically add vehicle in the road. 


\section{System Testing}

Software testing plays an increasingly important role in software engineering management, and the design of test cases is the basis of the entire testing process. The test case is not only the bridge between the test plan and the execution, but also the central content of the software test. Effective design of test cases, is the key to doing software testing. With the test file, and then refer to the test case can guarantee the quality of the test, in short, the test case is the guidance of the test work, software testing must comply with the rules, but also the quality of software testing is the fundamental guarantee. The test results are obvious, test cases comprehensive, each group of tests have reached the desired results. The system test case see table 1 .

Table 1 Test cases

\begin{tabular}{|c|r|}
\hline $\begin{array}{c}\text { Test function } \\
\text { points: }\end{array}$ & Functional testing of the software \\
\hline Test input: & Start road: 3 exit section: 2 road state: 0 switch to 1 \\
\hline Test Type: & $\begin{array}{r}\text { Functional testing, boundary testing, anomaly testing, performance testing, } \\
\text { start / stop testing, document testing }\end{array}$ \\
\hline Test operation: & $\begin{array}{r}\text { 1. Run the software 2. Initialize 3. Enter the data 4. Simulate the vehicle } \\
\text { into 5. Push the time and observe the way out }\end{array}$ \\
\hline expected & 1 state by 2 road exit 0 state when waiting in 3 road \\
\hline
\end{tabular}

\section{Conclusions}

Based on the basis of feasibility analysis and requirement analysis, the system develops an intelligent transportation simulation system under the environment of Microsoft Visual Studio 2010 and C\#.NET, with the features of clear structure, brief interface and convenient operation, etc. Users can obtain useful information conveniently.

The system is divided into five function modules: initialization module, state switch module, time propulsion module, vehicle simulation entering module and advanced control module. In addition, due to the limited design time, there are some deficiencies as well: functional requirements are not comprehensive, the incomplete interface, the less perfect code written, the less specific exception handling, etc. If users can report the errors to developers when using the system, and require maintenance and update, the application software can be as perfect as possible.

\section{Acknowledgement}

This work was supported by the Liaoning Provincial Social Planning Fund (L15BGL017) and science and technology program of Liaoning(20170540767).

\section{References}

[1] Wang G, Jiang Z, Qian J, et al. A web-based simulation system for traffic evacuation[C]// International Conference on Service Systems and Service Management. 2016:1-6.

[2] Yuan S, Chun S A, Spinelli B, et al. Traffic evacuation simulation based on multi-level driving decision model[J]. Transportation Research Part C Emerging Technologies, 2017, 78:129-149. 
[3] Yin W, Murray P. An agent-based modeling system for travel demand simulation for hurricane evacuation[J]. Transportation Research Part C Emerging Technologies, 2014, 42(42):44-59.

[4] Bakillah M, Zipf A, Liang S H L. Publish/Subscribe System Based on Event Calculus to Support Real-Time Multi-Agent Evacuation Simulation[M]// Geographic Information Science at the Heart of Europe. Springer International Publishing, 2013:337-352.

[5] Goetz M, Zipf A. Using Crowdsourced Geodata for Agent-Based Indoor Evacuation Simulations[J]. ISPRS International Journal of Geo-Information, 2012, 1(3):186-208.

[6] Naghawi H, Wolshon B. Performance of Multi-Modal Evacuation Traffic Networks: A Simulation Based Assessment[C]// Transportation Research Board 90th Annual Meeting. 2011.

[7] Yuan S, Liu Y, Wang G, et al. A Cross-Simulation Method for Large-Scale Traffic Evacuation with Big Data[M]// Web-Age Information Management. Springer International Publishing, 2014:14-21.

[8] Naghawi H, Wolshon B. Performance of Traffic Networks during Multimodal Evacuations: Simulation-Based Assessment[J]. Natural Hazards Review, 2012, 13(3):196-204.

[9] Yuan S, Liu Y, Wang G, et al. Research on meta-model of driver behavior in agent-based traffic evacuation simulation[J]. 2014:1805-1810. 